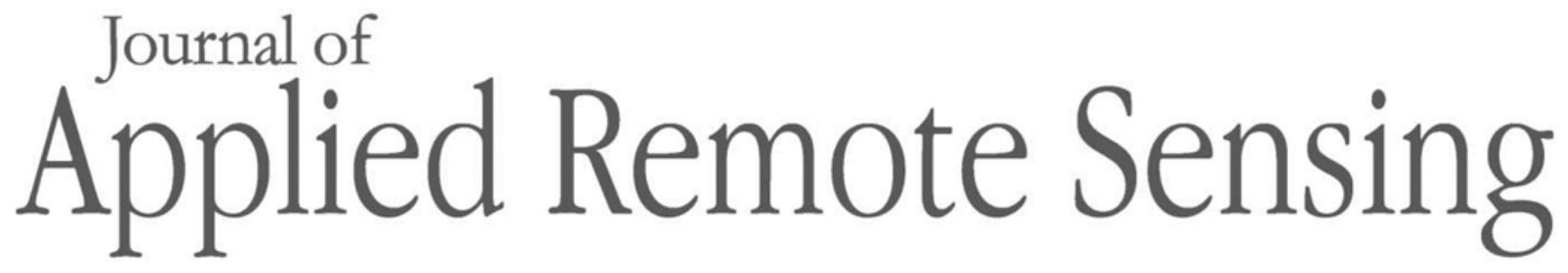

RemoteSensing.SPIEDigitalLibrary.org

\title{
Restoration of remote sensing images based on nonconvex constrained high-order total variation regularization
}

Jianguang Zhu

Kai Li

Binbin Hao 


\title{
Restoration of remote sensing images based on nonconvex constrained high-order total variation regularization
}

\author{
Jianguang Zhu, ${ }^{a} \mathrm{Kai} \mathrm{Li}{ }^{a}$ and Binbin $\mathrm{Hao}^{\mathrm{b}, *}$ \\ ${ }^{a}$ Shandong University of Science and Technology, College of Mathematics and \\ Systems Science, Qingdao, China \\ ${ }^{\mathrm{b}}$ China University of Petroleum, College of Science, Qingdao, China
}

\begin{abstract}
Convex total variation (TV) regularization models have been widely used in remote sensing image restoration problems; however, these models tend to produce staircase effects. We consider a nonconvex second-order TV regularization model with linear constraints for remote sensing image restoration. To solve the nonconvex second-order TV regularization model, we propose an efficient alternating minimization algorithm based on generalized iterated shrinkage algorithm and alternating direction method of multipliers. Experimental results demonstrate the effectiveness of the proposed model, which can reduce staircase effects while preserving edges. In terms of signal-to-noise ratio and structural similarity index measure, the experimental results show that our proposed model and algorithm can give better performance compared with some state-of-the-art methods. (C) 2019 Society of Photo-Optical Instrumentation Engineers (SPIE) [DOI: 10.1117/1.JRS.13.022006]
\end{abstract}

Keywords: remote sensing image; nonconvex; second-order total variation regularization; generalization of soft-thresholding algorithm.

Paper 180708SS received Aug. 29, 2018; accepted for publication Oct. 23, 2018; published online Jan. 2, 2019.

\section{Introduction}

Image restoration has been widely studied in remote sensing image processing in the last decades. ${ }^{1-6}$ Image restoration problem refers to recovering an image from blurry and noisy observation. For simplicity, we assume that the underlying images have square domains and are grayscale. Let $u \in R^{M \times M}$ be an original image, $K \in R^{M \times M}$ represent a blurring or convolution operator, $n \in R^{M \times M}$ be an additive noise, and $g \in R^{M \times M}$ be the degraded or contaminated image. The image restoration model can be described as follows:

$$
g=K u+n .
$$

It is well known that recovering $u$ from $g$ is a classical linear ill-posed inverse problem, and it is hard to directly find the solution. Many scholars have done a lot of research on the ill-posed problem and found that adding a regularization term to the restoration model can solve this problem effectively. Consequently, the image restoration methods with regularization have attracted wide attention.

A well-known regularized inverse problem is the Tikhonov regularization approach, ${ }^{7}$ which can be formulated as a one-step filter via Fourier transform for image restoration. Therefore, it produces a smoothing effect on the restored image, i.e., the Tikhonov-like regularization tends to make images overly smooth and often fails to preserve image edges. In comparison, a successful image restoration regularization model is the popular total variation (TV) restoration model, which was first proposed by Rudin et al. ${ }^{8,9}$ for Gaussian noise removal and then extended to image deconvolution. This regularization approach achieves an important advantage for edge-preserving image restoration. It has been proved to be effective both experimentally and theoretically. The model with TV regularization can be described as

*Address all correspondence to: Binbin Hao, E-mail: bbhao981@163.com

$1931-3195 / 2019 / \$ 25.00$ (C) 2019 SPIE 


$$
\min _{u}\|\nabla u\|_{1}+\frac{\mu}{2}\|K u-g\|_{2}^{2}
$$

The first term describes the TV regularization, where $\nabla u$ denotes the gradient of $u$, and it is defined as $\nabla u=\left(\nabla_{x} u, \nabla_{y} u\right)^{T}$. The second term is the fidelity term, which measures the difference between $g$ and $K u$. And $\mu>0$ is a regularized scale parameter tuning the weight between these two terms. $\nabla_{x}$ and $\nabla_{y}$ are the two linear differential operators given as

$$
\left(\nabla_{x} u\right)_{i, j}=\left\{\begin{array}{cl}
u_{i+1, j}-u_{i, j} & \text { if } i<M, \\
u_{1, j}-u_{M, j} & \text { if } i=M,
\end{array} \quad\left(\nabla_{y} u\right)_{i, j}=\left\{\begin{array}{cl}
u_{i, j+1}-u_{i, j} & \text { if } j<M \\
u_{i, 1}-u_{i, M} & \text { if } j=M
\end{array}\right.\right.
$$

for $i, j=1, \ldots, M$. Here, $u_{i, j}$ refers to the $(j M+i)^{\prime}$ th entry of the vector $u$. It is the $(i, j)^{\prime}$ th pixel location of the image, see Ref. 10.

The TV models have shown a remarkable advantage in preserving images' sharp edges. In the last decade, a number of methods have been proposed to solve the unconstrained model [Eq. (2)], such as a fixed point iteration method, Newton's method, Chambolle's projection algorithm, iterative shrinkage/thresholding algorithms, alternating direction minimization (ADM) methods (see for instance Refs. 11-28 and references therein). However, TV-based method suffers from the so-called staircasing phenomenon. Staircase solutions developed false edges that do not exist in the true image. To alleviate this drawback, many improved variation models have been proposed, such as high-order TV regularization methods ${ }^{29-31}$ and fractional order TV model. ${ }^{32-35}$ Combining the first-order and second-order TV regularizations, Papafitsoros and Schönlieb $^{36}$ proposed a hybrid variational model. By balancing the first- and second-order derivative regularizations, Bredies et al. ${ }^{37}$ proposed the total generalized variation (TGV) model, which can eliminate the staircase artifacts. In this paper, we focus on the high-order TV regularization. The majority of the high-order norms involve second-order differential operators because piecewise vanishing second-order derivatives lead to piecewise linear solutions that better fit smooth regions (see Ref. 38 for more details).

The above regularization terms lead to a convex optimization. It is well known that the convergence of the convex optimization problem is guaranteed. TV minimization, which is the $l_{1}$ norm of the gradient magnitude image (GMI), exploits the sparsity of GMI. However, the $l_{1}$ norm usually underestimates the nonzero values underlying the signal. ${ }^{39}$ Chen and Selesnick ${ }^{40}$ indicated that nonconvex regularizer can exhibit sparser solution than $l_{1}$ regularizer. To improve the shortcoming, a number of nonconvex regularizers are introduced. Nikolova et al. $^{41}$ developed a nonsmooth nonconvex image restoration model to recover image with neat edges. Based on wavelet tight frame and the TV, Lv et al. ${ }^{42}$ investigated a nonconvex hybrid variational regularization for restoring the degraded images. Using nonconvex and nonsmooth potential function, Zhang et al. ${ }^{43}$ proposed a nonconvex and nonsmooth TGV model. Recent research reveals that for modeling the sparseness of image gradient, the $l_{p}$-norm $\left(\|\cdot\|_{p}^{p}\right)$ with $0<p<1$ is more suitable than the $l_{1}$-norm $\left(\|\cdot\|_{1}\right)$ of TV regularizer. ${ }^{44}$ In the works of $\mathrm{Xu}$ et al., ${ }^{45}$ an efficient iterative half-thresholding algorithm to solve the $l_{\frac{1}{2}}$ norm for noisy signal recovery was proposed. In Ref. 46, Zuo et al. introduced a generalized iterated shrinkage algorithm (GISA) by extending the popular soft-thresholding operator to solve the following image deconvolution model with $p$-norm:

$$
\min _{u} \mu\|\nabla u\|_{p}^{p}+\frac{1}{2}\|K u-g\|_{2}^{2}
$$

Recently, Afonso et al. ${ }^{47}$ proposed the following constrained TV regularized problem:

$$
\min _{u} \phi(u) \quad \text { s.t. }\|K u-g\| \leq \delta
$$

where the parameter $\delta>0$ is an estimate of the noise level in the data and $\phi(u)$ is a regularization function. In the case where $\phi(u)=\|u\|_{1}$, the above problem is usually known as basis pursuit denoising (BPD). ${ }^{48}$ Meanwhile, the authors put forward a constrained split augmented Lagrangian shrinkage algorithm (C-SALSA) to solve the constrained model [Eq. (4)]. The 
experimental results indicate that C-SALSA method is effective and promising. Constrained problems are usually much more difficult to solve than unconstrained ones. Although, it has the important advantage that choosing a reasonable parameter $\delta$ is easier than finding a suitable regularization parameter $\mu^{49}$

Inspired by the above-mentioned advantages of the nonconvex regularization and secondorder TV regularization, we propose the following nonconvex approximation model with a linear constraint:

$$
\min _{u}\left\|\nabla^{2} u\right\|_{p}^{p} \quad \text { s.t. }\|K u-g\|_{2} \leq \delta
$$

where $\nabla^{2} u=\left(\begin{array}{cc}\nabla_{x x} u & \nabla_{x y} u \\ \nabla_{y x} u & \nabla_{y y} u\end{array}\right)$ denotes the second-order discrete gradient of $u$. For solving the proposed nonconvex model [Eq. (5)], combining generalization of soft-thresholding algorithm and alternating direction method, we develop an efficient alternating iterated algorithm. The detailed solution process will be explained in Sec. 2. We report experimental results and do some comparisons. The comparison results show that our method is efficient and performs better than some state-of-the-art methods.

The paper is organized as follows: in Sec. 2, using the variable splitting technique, augmented Lagrangian method of multipliers (ADMM), and generalized soft-thresholding algorithm, an efficient alternating iterated algorithm is proposed to solve the proposed model [Eq. (5)]. In Sec. 3, we present numerical results and performance comparisons. Finally, Sec. 4 concludes this paper.

\section{Solving Constrained Nonconvex Second-Order Total Variation Image Restoration Model}

In this section, we propose an efficient method to solve the nonconvex constrained second-order TV [Eq. (5)]. Based on variable splitting technology and generalized soft-thresholding function, ADMM is used to solve the proposed nonconvex constrained second-order TV model [Eq. (5)].

By introducing two auxiliary variables $\omega$ and $r$, we can obtain the following equivalent form of the model [Eq. (5)]:

$$
\begin{aligned}
& \min _{u, \omega}\|\omega\|_{p}^{p} \quad \text { s.t. } \omega=\nabla^{2} u, \\
& K u-g=r\left(\|r\|_{2} \leq \delta\right) .
\end{aligned}
$$

To further translate the above-constrained problem into unconstrained ones, the augmented Lagrangian function is introduced. The augmented Lagrangian function of Eq. (6) is defined as follows:

$$
\begin{aligned}
L_{A}\left(\omega, u, \lambda_{1}, \lambda_{2}\right)= & \|\omega\|_{p}^{p}-\lambda_{1}^{T}\left(\omega-\nabla^{2} u\right)+\frac{\beta_{1}}{2}\left\|\omega-\nabla^{2} u\right\|_{2}^{2} \\
& -\lambda_{2}^{T}(K u-g-r)+\frac{\beta_{2}}{2}\|K u-g-r\|_{2}^{2},
\end{aligned}
$$

where $\lambda_{1}$ and $\lambda_{2}$ are the Lagrange multipliers, $\beta_{1}$ and $\beta_{2}$ are the penalty parameters.

According to the idea of classical ADMM, the solution of the problem [Eq. (7)] is to find a saddle point of $L_{A}\left(\omega, u, \lambda_{1}, \lambda_{2}\right)$. This can be done by alternately minimizing the augmented Lagrangian function $L_{A}(\cdot)$ with the following form:

$$
\left\{\begin{array}{l}
\omega^{k+1}=\arg \min _{\omega} L_{A}\left(\omega, u^{k}, \lambda_{1}^{k}, \lambda_{2}^{k}\right), \\
u^{k+1}=\arg \min _{u} L_{A}\left(\omega^{k+1}, u, \lambda_{1}^{k}, \lambda_{2}^{k}\right), \\
r^{k+1}=\arg \min _{r}-\left(\lambda_{2}^{k}\right)^{T}(K u-g-r)+\frac{\beta_{2}}{2}\left\|K u^{k+1}-g-r\right\|_{2}^{2}, \\
\text { s.t. }\|r\|_{2} \leq \delta,
\end{array}\right.
$$


Zhu, Li, and Hao: Restoration of remote sensing images based on nonconvex constrained high-order...

and the Lagrange multiplier parameters are updated as follows:

$$
\left\{\begin{array}{l}
\lambda_{1}^{k+1}=\lambda_{1}^{k}-\beta_{1} \xi\left(\omega^{k+1}-\nabla^{2} u^{k+1}\right), \\
\lambda_{2}^{k+1}=\lambda_{2}^{k}-\beta_{2} \xi\left(K u^{k+1}-g-r^{k+1}\right),
\end{array}\right.
$$

where $\xi$ is a relaxation parameter. Next, we investigate the subproblems one by one.

(1) The $\omega$ subproblem: The $\omega$ subproblem is a nonconvex minimization problem due to the nonconvex $l_{p}$ norm regularizer. For fixed $u^{k}, \lambda_{1}^{k}$, and $\lambda_{2}^{k}$, the minimization of Eq. (7) with respect to $\omega$ can be obtained as

$$
\begin{aligned}
\omega^{k+1} & =\arg \min _{\omega} L_{A}\left(\omega, u^{k}, \lambda_{1}^{k}, \lambda_{2}^{k}\right) \\
& =\arg \min _{\omega}\|\omega\|_{p}^{p}-\left(\lambda_{1}^{k}\right)^{T}\left(\omega-\nabla^{2} u^{k}\right)+\frac{\beta_{1}}{2}\left\|\omega-\nabla^{2} u^{k}\right\|_{2}^{2} \\
& =\arg \min _{\omega}\|\omega\|_{p}^{p}+\frac{\beta_{1}}{2}\left\|\omega-\left(\nabla^{2} u^{k}+\frac{\lambda_{1}^{k}}{\beta_{1}}\right)\right\|_{2}^{2} .
\end{aligned}
$$

There are a number of methods proposed to solve the above $\omega$ subproblem [Eq. (8)], such as iteratively reweighted $l_{1}$-minimization and iteratively reweighted least squares method. ${ }^{50-53}$ However, these methods could not converge to the global optimal solution. To guarantee the convergence of minimization of $\omega$ subproblem, Zuo et al. ${ }^{46}$ employed a generalized soft thresholding algorithm (GST) to solve this problem. Then, the solutions $\omega^{k+1}$ are given as

$$
\omega^{k+1}=T_{p}^{\mathrm{GST}}\left(\nabla^{2} u^{k}+\frac{\lambda_{1}^{k}}{\beta_{1}} ; \frac{1}{\beta_{1}}\right) .
$$

The function $T_{p}^{\mathrm{GST}}$ in Eq. (9) is defined as

$$
T_{p}^{\mathrm{GST}}(y ; \lambda)= \begin{cases}0, & \text { if }|y| \leq \tau_{p}^{\mathrm{GST}}(\lambda), \\ \operatorname{sgn}(y) S_{p}^{j}(|y| ; \lambda), & \text { if }|y|>\tau_{p}^{\mathrm{GST}}(\lambda),\end{cases}
$$

where $\operatorname{sgn}(\cdot)$ is the signum function, $S_{p}^{j+1}(|y| ; \lambda)$ is iteratively computed by the following equation:

$$
S_{p}^{j+1}(|y| ; \lambda)=|y|-\lambda p\left[S_{p}^{j}(|y| ; \lambda)\right]^{p-1}, \quad j=0,1, \ldots, J,
$$

$S_{p}^{0}(|y| ; \lambda)=|y|$, and the thresholding value $\tau_{p}^{\mathrm{GST}}(\lambda)$ is defined as follows:

$$
\tau_{p}^{G S T}(\lambda)=[2 \lambda(1-p)]^{\frac{1}{2-p}}+\lambda p[2 \lambda(1-p)]^{\frac{p-1}{2-p}} .
$$

(2) The $u$ subproblem: The minimization of subproblem with $u$ can be solved as

$$
\begin{aligned}
u^{k+1}= & \arg \min _{u} L_{A}\left(\omega^{k+1}, u, \lambda_{1}^{k}, \lambda_{2}^{k}\right) \\
= & \arg \min _{u}-\left(\lambda_{1}^{k}\right)^{T}\left(\omega^{k+1}-\nabla^{2} u\right)+\frac{\beta_{1}}{2}\left\|\omega^{k+1}-\nabla^{2} u\right\|_{2}^{2} \\
& -\left(\lambda_{2}^{k}\right)^{T}\left(K u-g-r^{k}\right)+\frac{\beta_{2}}{2}\left\|K u-g-r^{k}\right\|_{2}^{2} \\
= & \arg \min _{u} \frac{\beta_{1}}{2}\left\|\omega^{k+1}-\left(\nabla^{2} u+\frac{\lambda_{1}^{k}}{\beta_{1}}\right)\right\|_{2}^{2}+\frac{\beta_{2}}{2}\left\|K u-\left(g+r^{k}+\frac{\lambda_{2}^{k}}{\beta_{2}}\right)\right\|_{2}^{2} .
\end{aligned}
$$

Then, we can obtain the first-order necessary optimality conditions of Eq. (10) as follows:

$$
\left(\beta_{1} \nabla^{2 T} \nabla^{2}+\beta_{2} K^{T} K\right) u=\beta_{1} \nabla^{2 T} \omega^{k+1}-\nabla^{2 T} \lambda_{1}^{k}+\beta_{2} K^{T}\left(g+r^{k}+\frac{\lambda_{2}^{k}}{\beta_{2}}\right) .
$$


Zhu, Li, and Hao: Restoration of remote sensing images based on nonconvex constrained high-order...

Under the periodic boundary condition for $u, \nabla^{2 T} \nabla^{2}$ and $K^{T} K$ are all block circulant matrices, more details see Ref. 54, and the matrices can be diagonalized by the two-dimensional (2-D) fast discrete Fourier transforms. ${ }^{55}$ So, the solution of Eq. (11) can be obtained by two fast discrete Fourier transforms and the solution has the following closed form:

$$
u^{k+1}=\mathcal{F}^{-1}\left[\frac{\mathcal{F}\left(\beta_{1} \nabla^{2 T} \omega^{k+1}-\nabla^{2 T} \lambda_{1}^{k}+\beta_{2} K^{T}\left(g+r^{k}\right)+K^{T} \lambda_{2}^{k}\right)}{\mathcal{F}\left(\beta_{1} \nabla^{2 T} \nabla^{2}+\beta_{2} K^{T} K\right)}\right] .
$$

(3) The $r$ subproblem: The $r$ subproblem is equivalently transformed to the following form:

$$
r^{k+1}=\arg \min _{r \in \Omega} \frac{\beta_{2}}{2}\left\|K u^{k+1}-g-r-\frac{\lambda_{2}^{k}}{\beta_{2}}\right\|_{2}^{2},
$$

where $\Omega=\left\{r \in \mathbf{R}^{M} \mid\|r\|_{2} \leq \delta\right\}$. The above minimization can be directly obtained by the following projection:

$$
r^{k+1}=\mathcal{P}_{\Omega}\left[K u^{k+1}-g-r-\frac{\lambda_{2}^{k}}{\beta_{2}}\right]
$$

where $\mathcal{P}_{\Omega}$ denotes the projection operator.

Finally, we update the Lagrange multipliers $\lambda_{1}$ and $\lambda_{2}$ as

$$
\begin{gathered}
\lambda_{1}^{k+1}=\lambda_{1}^{k}-\beta_{1} \xi\left(\omega^{k+1}-\nabla^{2} u^{k+1}\right), \\
\lambda_{2}^{k+1}=\lambda_{2}^{k}-\beta_{2} \xi\left(K u^{k+1}-g-r^{k+1}\right) .
\end{gathered}
$$

The parameter $\xi$ in Eqs. (14) and (15) is a relaxation parameter. It is well known that when $\xi \in[0,(\sqrt{5}+1) / 2]$, the algorithm has the best convergence. In this paper, we select $\xi=0.55$.

We name the proposed algorithm as the nonconvex constrained high-order TV with alternating direction method of multipliers (abbreviated as NCHTV-ADMM), which is presented in Algorithm 1.

\section{Numerical Experiments}

In this section, we present some numerical examples of image restoration to illustrate the effectiveness of our proposed NCHTV model. We test several remote sensing images including Aerial (1) $(256 \times 256)$, chemical plant $(256 \times 256)$, and Aerial(2) $(512 \times 512)$. The three different types of images are shown in Fig. 1. The experiments are performed under Windows 10 with MATLAB version 2012a running on a PC with an Intel Core i5Duo Central processing unit at $2.50 \mathrm{GHz}$ and $4 \mathrm{~GB}$ of memory.

The signal-to-noise ratio (SNR), structural similarity index measure (SSIM), and relative error $\left(R_{\text {err }}\right)^{56}$ are used to compare the quality of the restoration results. They are defined as follows:

$$
\begin{gathered}
\mathrm{SNR}=20 \log _{10} \frac{\left\|u^{0}-\bar{u}\right\|_{2}}{\left\|u^{0}-u\right\|_{2}}, \\
\mathrm{SSIM}=\frac{\left(2 \mu_{u^{0}} \mu_{u}+c_{1}\right)\left(2 \sigma_{u^{0} u}+c_{2}\right)}{\left(\mu_{u^{0}}^{2}+\mu_{u}^{2}+c_{1}\right)\left(\sigma_{u^{0}}^{2}+\sigma_{u}^{2}+c_{2}\right)}, \\
R_{\mathrm{err}}=\frac{\left\|u-u^{0}\right\|_{2}}{\left\|u^{0}\right\|_{2}},
\end{gathered}
$$


Algorithm 1 NCHTV with ADMM.

1. Input: $g, K, \beta_{1}>0, \beta_{2}>0, p, J$

2. Initialization: $u^{0}=g, \omega^{0}=\nabla^{2} u^{0}, \xi=0.55, \lambda_{i}=0$ for $i=1,2$.

3. While "not converged," Do

4. Compute $\omega^{k+1}$

$$
\omega^{k+1}=T_{p}^{G S T}\left(\nabla^{2} u^{k}+\frac{\lambda_{1}^{k}}{\beta_{1}} ; \frac{1}{\beta_{1}}\right)
$$

5. Compute $u^{k+1}$ via

$$
u^{k+1}=\mathcal{F}^{-1}\left[\frac{\mathcal{F}\left(\beta_{1} \nabla^{2 T} \omega^{k+1}-\nabla^{2 T} \lambda_{1}^{k}+\beta_{2} K^{T}\left(g+r^{k}\right)+K^{T} \lambda_{2}^{k}\right)}{\mathcal{F}\left(\beta_{1} \nabla^{2 T} \nabla^{2}+\beta_{2} K^{T} K\right)}\right]
$$

6. Compute $r^{k}$ by

$$
r^{k+1}=\mathcal{P}_{\Omega}\left[K u^{k+1}-g-r-\frac{\lambda_{2}^{k}}{\beta_{2}}\right]
$$

7. Update $\lambda_{1}^{k+1}$

$$
\lambda_{1}^{k+1}=\lambda_{1}^{k}-\beta_{1} \xi\left(\omega^{k+1}-\nabla^{2} u^{k+1}\right)
$$

8. Update $\lambda_{2}^{k+1}$

$$
\lambda_{2}^{k+1}=\lambda_{2}^{k}-\beta_{2} \xi\left(K u^{k+1}-g-r^{k+1}\right)
$$

\section{End Do}

10. Output $u^{k+1}$

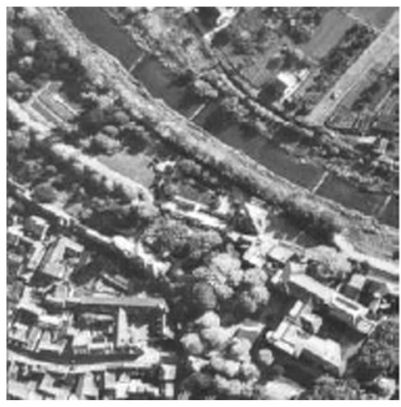

(a)

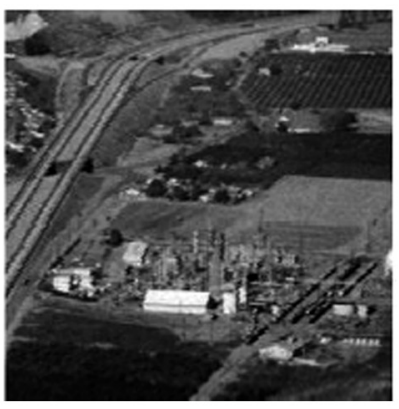

(b)

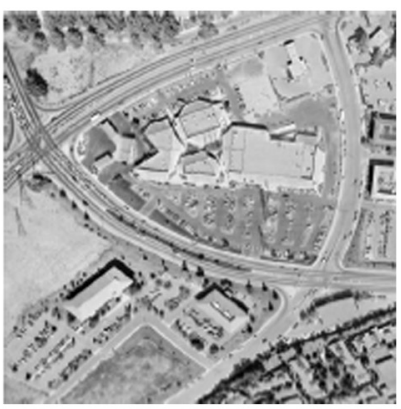

(c)

Fig. 1 Test images used for the experiments: (a) Aerial(1), (b) chemical plant, and (c) Aerial(2).

where $u^{0}, u$ are the original image and the restored image, respectively, $\bar{u}$ is the mean intensity value of $u^{0} \cdot \mu_{u^{0}}$ and $\mu_{u}$ are the mean values of the $u^{0}$ and $u$, respectively, $\sigma_{u^{0}}^{2}$ and $\sigma_{u}^{2}$ represent the variance of the $u^{0}$ and $u$, respectively, and $\sigma_{u^{0} u}$ is the covariance of the $u^{0}$ and $u, c_{1}$ and $c_{2}$ are the positive constants that can be seen as stabilizing constants for near-zero denominator values. Generally, the larger SNR values show that the restored images are better. The SSIM is an index that is used to measure the similarity between the restored image and the ideal image. The closer the values of SSIM are to 1 , the closer the restored image is to the original ones. 
Zhu, Li, and Hao: Restoration of remote sensing images based on nonconvex constrained high-order...

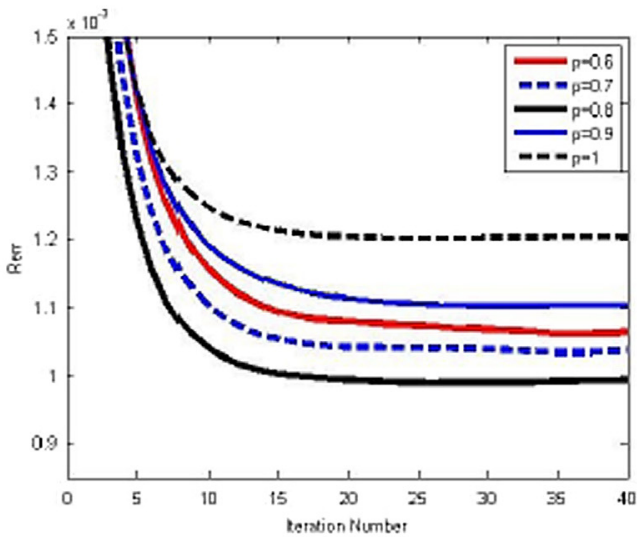

(a) $\delta=0.02$

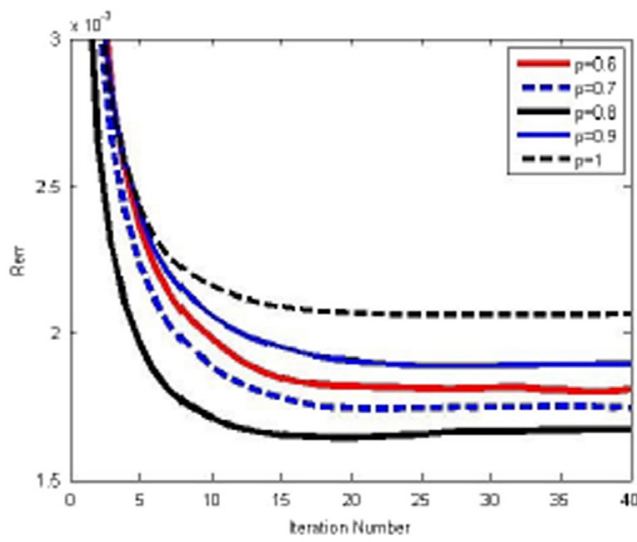

(c) $\delta=0.02$

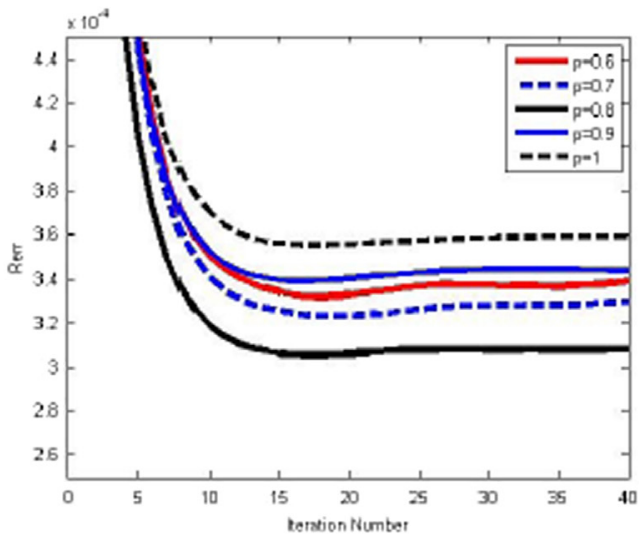

(e) $\delta=0.02$

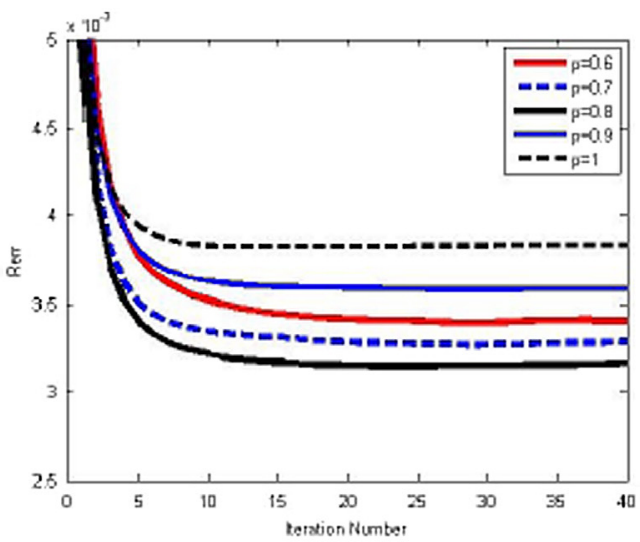

(b) $\delta=0.1$

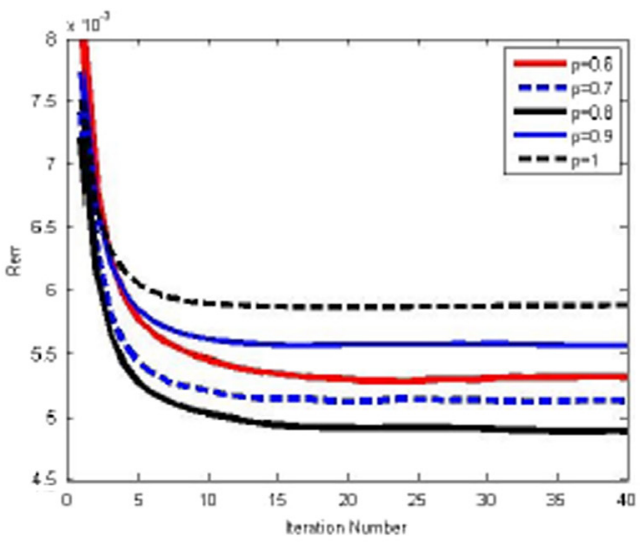

(d) $\delta=0.1$

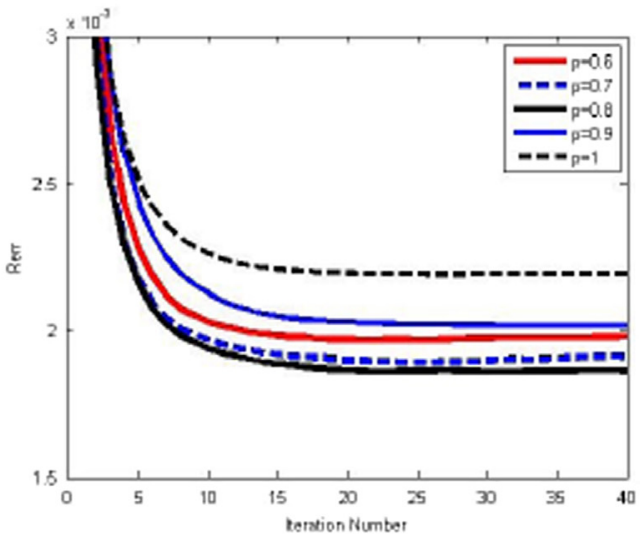

(f) $\delta=0.1$

Fig. $2 R_{\text {err }}$ values versus iteration with different $p$. (a) and (b) Corrupted by Gaussian blur. (c) and (d) Corrupted by average blur. (e) and (f) Corrupted by motion blur.

And, the smaller the $R_{\text {err }}$ values are, then the better the performance is. The stopping criterion of the testing algorithms in all the experiments is set as follows:

$$
\frac{\left\|u^{k+1}-u^{k}\right\|_{2}}{\left\|u^{k}\right\|_{2}} \leq 10^{-4} .
$$

We compare the proposed method with two related methods: one is the GISA, which was proposed by Zuo et al. ${ }^{46}$ to solve the nonconvex $l_{p}$ regularization image restoration; the other is a fast TV regularization based method with alternating direction method of multipliers (FTVd). ${ }^{28}$ 


\subsection{Experiment 1}

In this experiment, we show the effect of parameter $p$ to the recovery performance. We test the proposed NCHTV-ADMM for restoring the image "chemical plant" with different values of $p$ under different blurring kernels and different noise levels. In Fig. 2, we plot the $R_{\text {err }}$ behaviors along with associated iteration numbers under different values of $p$. It can be observed from Fig. 2 that the proposed NCHTV-ADMM generates decreasing sequences when $p=1,0.9,0.6,0.7,0.8$. From this experiment, it is clear that NCHTV-ADMM performs better when $p=0.8$, and we set $p=0.8$ in the following experiments.

\subsection{Experiment 2}

In this subsection, we perform some experiments to illustrate the performance of the proposed NCHTV-ADMM algorithm. To show the performance of the proposed NCHTV-ADMM, we compared it with two state-of-the-art methods, FTVd ${ }^{28}$ and GISA. ${ }^{46}$

First, the "Aerial(1)" images are degraded by Gaussian blurring operator. For an experiment with noise levels $\delta=0.02,0.1$ and Gaussian blur with Gaussian [Eq. (5)] kernels of size 11, Fig. 3 shows the restored results with FTVd, ${ }^{28}$ GISA, ${ }^{46}$ and the proposed NCHTV-ADMM. The zoomed parts of the restored images are shown in Fig. 4. For a more complete explanation, we also perform the experiments for the three tested images under different Gaussian blurring kernels. The corresponding detailed results of SNR and SSIM values are shown in Table 1. In Fig. 3, Fig. 4, and Table 1, the proposed algorithm demonstrates improvement in the restored images using our algorithm. Meanwhile, one can see that the proposed method can obtain better restoration results with higher SNRs and SSIMs.

Next, the average blur is considered. For an experiment with noise levels $\delta=0.02,0.1$ and average blur kernel of size $15 \times 15$, Fig. 5 shows the results obtained by FTVd, ${ }^{28}$ GISA, ${ }^{46}$ and the proposed NCHTV-ADMM algorithm. The zoomed parts of the restored images are shown in Fig. 6. We can easily see the proposed algorithm yields better results in image restoration as it avoids the staircase effect while preserving edges well. Table 2 shows the results of SNR and SSIM values under different average blurring kernels.

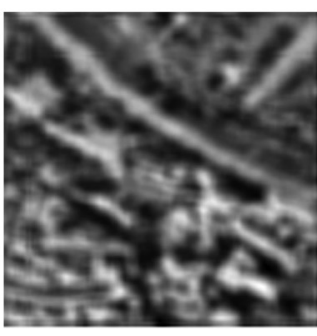

(a)

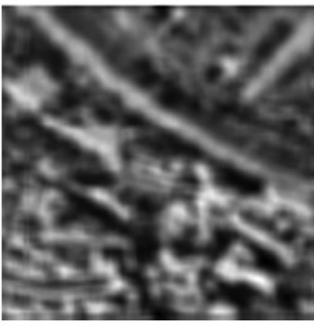

(e)

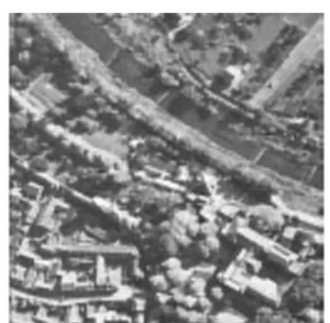

(b)

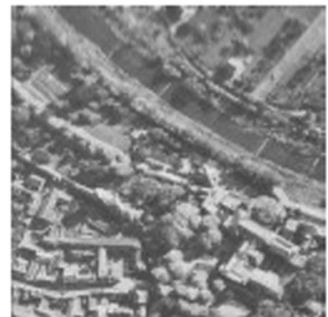

(f)

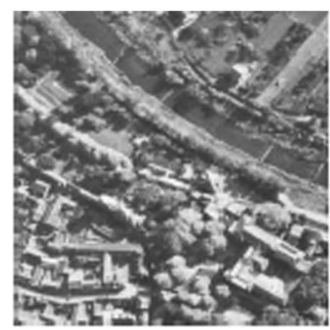

(c)

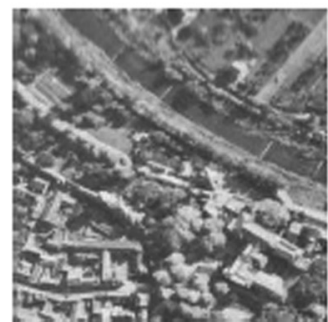

(g)

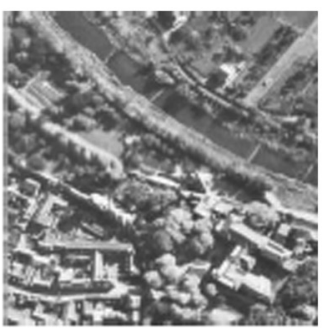

(d)

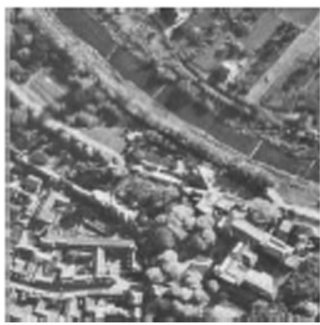

(h)

Fig. 3 Results of noisy images restored with different methods under $11 * 11$ Gaussian blur, with noise level $(\mathrm{a}-\mathrm{d}) \delta=0.02$ and $(\mathrm{e}-\mathrm{h}) \delta=0.1$. Columns from the left to the right in each row are the blurred noisy image, the restored image by FTVd, the restored image by GISA, the restored image by NCHTV-ADMM, respectively. 


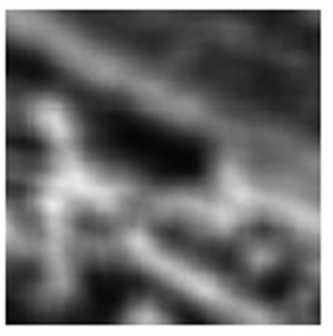

(a)

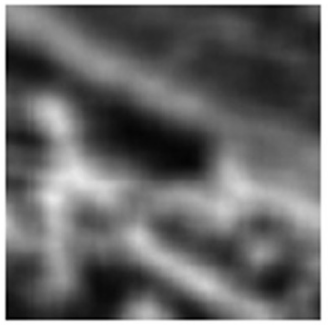

(e)

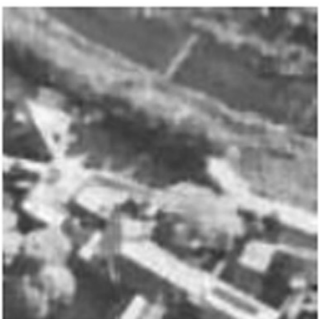

(b)

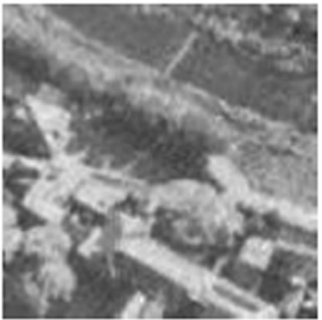

(f)

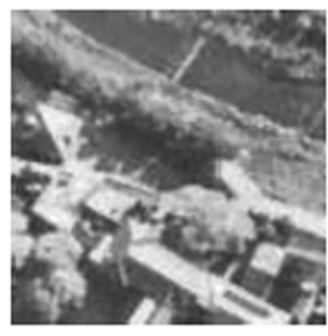

(c)

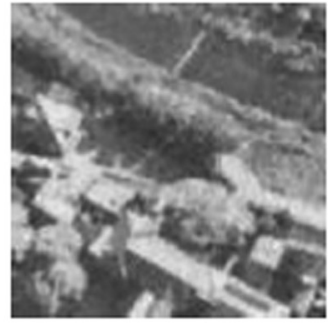

(g)

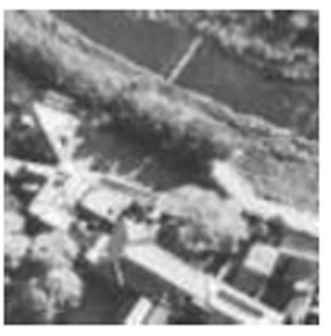

(d)

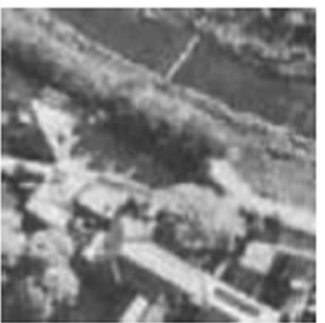

(h)

Fig. 4 Zoomed partial regions in Fig. 3.

Table 1 The restored results by FTVd, GISA, and NCHTV-ADMM for different images under Gaussian blur.

\begin{tabular}{|c|c|c|c|c|c|c|c|c|}
\hline \multirow{3}{*}{$\frac{\text { Blurring kernel }}{\text { Gaussian }\left(9^{*} 9\right)}$} & \multirow{2}{*}{\multicolumn{2}{|c|}{ Image }} & \multicolumn{3}{|c|}{$\delta=0.02$} & \multicolumn{3}{|c|}{$\delta=0.1$} \\
\hline & & & \multirow{2}{*}{$\frac{\text { FTVd }}{29.46}$} & \multirow{2}{*}{$\frac{\text { GISA }}{30.43}$} & \multirow{2}{*}{$\frac{\text { Our method }}{31.14}$} & \multirow{2}{*}{$\frac{\text { FTVd }}{24.23}$} & \multirow{2}{*}{$\frac{\text { GISA }}{25.54}$} & \multirow{2}{*}{$\frac{\text { Our method }}{26.36}$} \\
\hline & Aerial(1) & SNR & & & & & & \\
\hline & & SSIM & 0.9539 & 0.9608 & 0.9767 & 0.8762 & 0.8938 & 0.9186 \\
\hline & Chemical plant & SNR & 27.68 & 28.44 & 29.42 & 21.93 & 22.57 & 23.96 \\
\hline & & SSIM & 0.9214 & 0.9310 & 0.9440 & 0.7729 & 0.8309 & 0.8676 \\
\hline & Aerial(2) & SNR & 33.23 & 34.50 & 36.09 & 27.11 & 28.83 & 29.75 \\
\hline & & SSIM & 0.9347 & 0.9400 & 0.9549 & 0.7624 & 0.8299 & 0.8744 \\
\hline \multirow[t]{6}{*}{ Gaussian(11*11) } & Aerial(1) & SNR & 27.61 & 28.62 & 29.40 & 23.11 & 24.34 & 25.10 \\
\hline & & SSIM & 0.9313 & 0.9426 & 0.9530 & 0.8492 & 0.8792 & 0.8959 \\
\hline & Chemical plant & SNR & 25.95 & 26.77 & 27.79 & 20.83 & 22.48 & 23.71 \\
\hline & & SSIM & 0.8830 & 0.8998 & 0.9200 & 0.7287 & 0.7902 & 0.8281 \\
\hline & Aerial(2) & SNR & 31.76 & 32.96 & 4.41 & 26.01 & 27.83 & 29.08 \\
\hline & & SSIM & 0.9120 & 0.9210 & 0.9403 & 0.7426 & 0.8083 & 0.8532 \\
\hline \multirow[t]{6}{*}{ Gaussian(15*15) } & Aerial(1) & SNR & 25.33 & 26.42 & 27.23 & 21.70 & 22.86 & 23.35 \\
\hline & & SSIM & 0.8936 & 0.9129 & 0.9300 & 0.8103 & 0.8307 & 0.8542 \\
\hline & Chemical plant & SNR & 24.22 & 25.07 & 26.13 & 19.72 & 21.46 & 22.49 \\
\hline & & SSIM & 0.8353 & 0.8590 & 0.8891 & 0.6844 & 0.7514 & 0.7868 \\
\hline & Aerial(2) & SNR & 28.74 & 30.11 & 31.34 & 24.44 & 25.10 & 26.23 \\
\hline & & SSIM & 0.8529 & 0.8718 & 0.9001 & 0.6838 & 0.7461 & 0.7910 \\
\hline
\end{tabular}




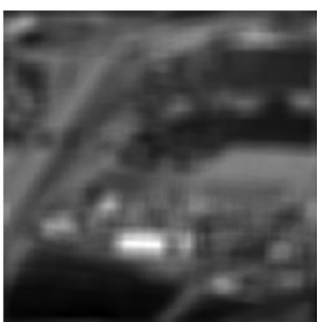

(a)

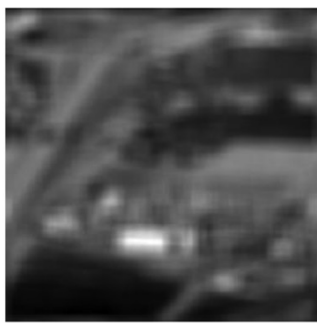

(e)

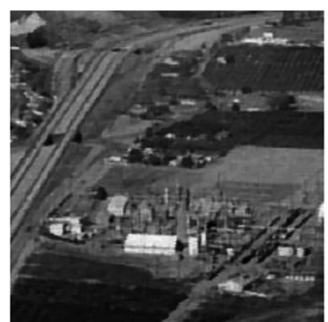

(b)

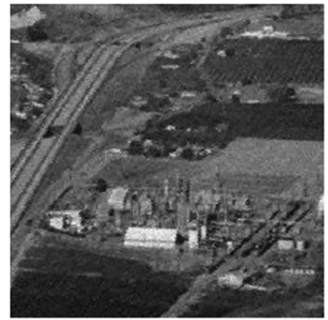

(f)

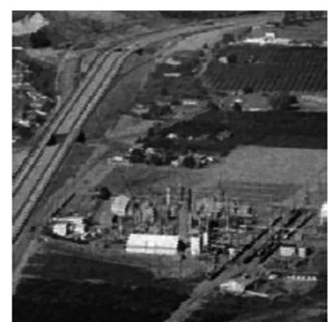

(c)

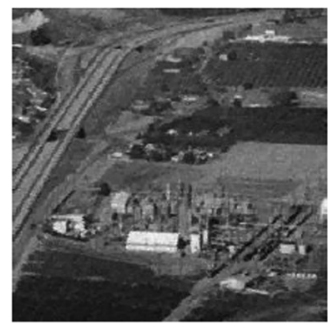

(g)

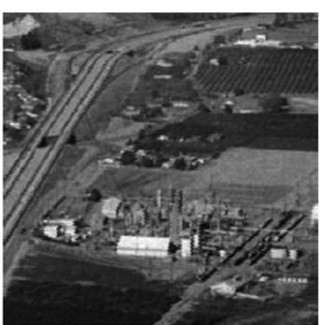

(d)

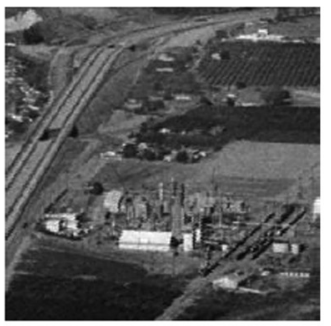

(h)

Fig. 5 Results of noisy images restored by different methods under $15 * 15$ average blur, with noise level $(\mathrm{a}-\mathrm{d}) \delta=0.02$ and $(\mathrm{e}-\mathrm{h}) \delta=0.1$. Columns from the left to the right in each row are the blurred noisy image, the restored image by FTVd, the restored image by GISA, and the restored image by NCHTV-ADMM, respectively.

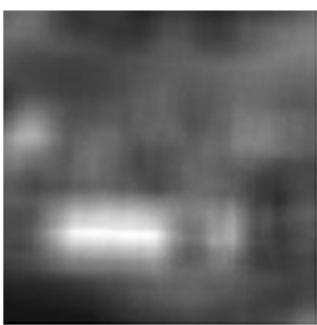

(a)

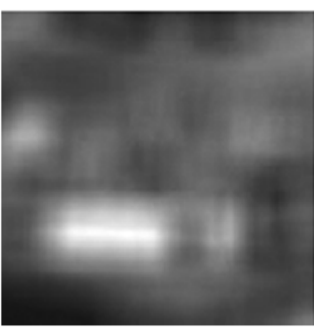

(e)

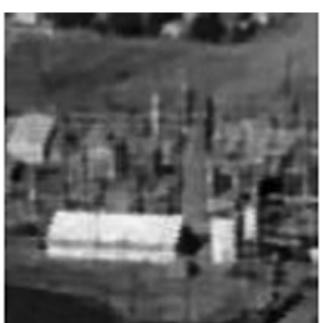

(b)

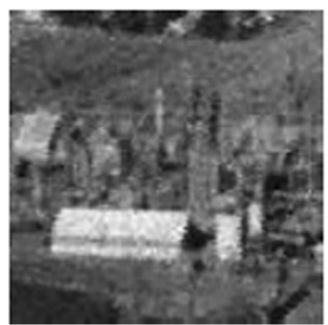

(f)

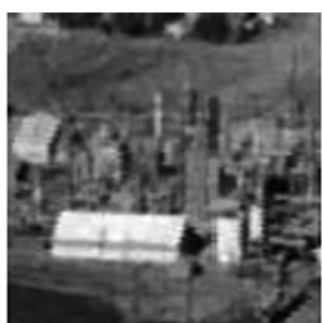

(c)

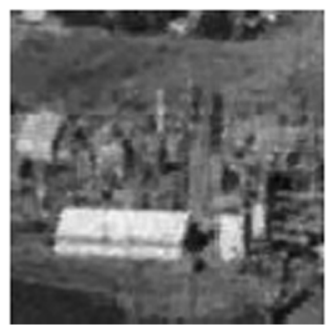

(g)

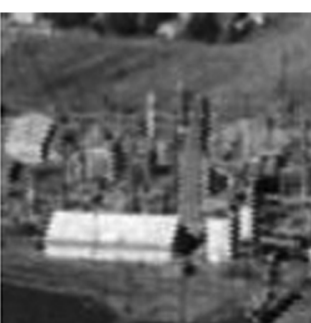

(d)

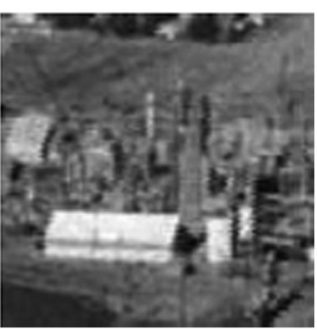

(h)

Fig. 6 Zoomed partial regions in Fig. 5. For a better visualization, some small partial regions of the restored results in Fig. 5 are zoomed.

Then, the ideal image "Aerial(2)" is degraded by a linear motion blur. For the experiment with noise levels $\delta=0.02,0.1$ and the motion kernels of length 55, Fig. 7 shows the results obtained by the above-mentioned three algorithms. For a better visualization, some small partial regions of the restored results of Fig. 7 are zoomed in Fig. 8. The results of SNR and SSIM values for tested images under different motion blurring kernels are shown in Table 3. Clearly, the visual quality of the restored image by the proposed NCHTV-ADMM algorithm is competitive with the other two algorithms. Moreover, one can observe that the SNRs and the SSIMs of the restored images by the proposed algorithm are better than those by the other two mentioned algorithms. 
Zhu, Li, and Hao: Restoration of remote sensing images based on nonconvex constrained high-order...

Table 2 The restored results by FTVd, GISA, and NCHTV-ADMM for different images under average blur.

\begin{tabular}{|c|c|c|c|c|c|c|c|c|}
\hline \multirow{3}{*}{$\frac{\text { Blurring kernel }}{\text { Average(9) }}$} & \multirow{2}{*}{\multicolumn{2}{|c|}{ Image }} & \multicolumn{3}{|c|}{$\delta=0.02$} & \multicolumn{3}{|c|}{$\delta=0.1$} \\
\hline & & & \multirow{2}{*}{$\frac{\text { FTVd }}{30.14}$} & \multirow{2}{*}{$\frac{\text { GISA }}{31.10}$} & \multirow{2}{*}{$\frac{\text { Our method }}{31.91}$} & \multirow{2}{*}{$\frac{\text { FTVd }}{24.73}$} & \multirow{2}{*}{$\frac{\text { GISA }}{25.94}$} & \multirow{2}{*}{$\frac{\text { Our method }}{26.87}$} \\
\hline & Aerial(1) & SNR & & & & & & \\
\hline & & SSIM & 0.9595 & 0.9659 & 0.9713 & 0.8854 & 0.9107 & 0.9256 \\
\hline & Chemical plant & SNR & 28.29 & 29.04 & 30.08 & 22.27 & 23.86 & 25.29 \\
\hline & & SSIM & 0.9300 & 0.9371 & 0.9512 & 0.7826 & 0.8377 & 0.8763 \\
\hline & Aerial(2) & SNR & 33.81 & 35.07 & 36.67 & 27.42 & 29.16 & 30.66 \\
\hline & & SSIM & 0.9383 & 0.9432 & 0.9566 & 0.7695 & 0.8338 & 0.8775 \\
\hline \multirow[t]{6}{*}{ Average(11) } & Aerial(1) & SNR & 28.36 & 29.48 & 30.36 & 23.47 & 24.78 & 25.53 \\
\hline & & SSIM & 0.9409 & 0.9518 & 0.9615 & 0.8572 & 0.8885 & 0.9036 \\
\hline & Chemical plant & SNR & 26.54 & 27.30 & 28.61 & 21.19 & 22.87 & 24.14 \\
\hline & & SSIM & 0.8965 & 0.9097 & 0.9333 & 0.7647 & 0.8058 & 0.8433 \\
\hline & Aerial(2) & SNR & 32.37 & 33.52 & 35.06 & 26.30 & 28.10 & 29.47 \\
\hline & & SSIM & 0.9203 & 0.9260 & 0.9450 & 0.7426 & 0.8100 & 0.8579 \\
\hline \multirow[t]{6}{*}{ Average(15) } & Aerial(1) & SNR & 25.25 & 26.32 & 27.08 & 22.00 & 22.86 & 23.25 \\
\hline & & SSIM & 0.8918 & 0.9119 & 0.9265 & 0.8153 & 0.8367 & 0.8496 \\
\hline & Chemical plant & SNR & 24.32 & 25.07 & 26.08 & 20.63 & 21.46 & 22.49 \\
\hline & & SSIM & 0.8379 & 0.8586 & 0.8876 & 0.7214 & 0.7504 & 0.7878 \\
\hline & Aerial(2) & SNR & 29.43 & 30.86 & 32.27 & 24.74 & 26.47 & 27.35 \\
\hline & & SSIM & 0.8666 & 0.8842 & 0.9145 & 0.6921 & 0.7352 & 0.7831 \\
\hline
\end{tabular}

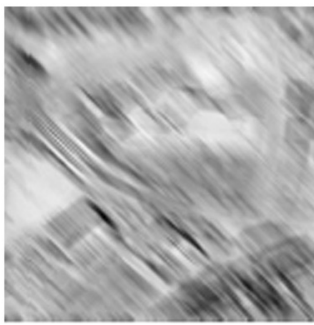

(a)

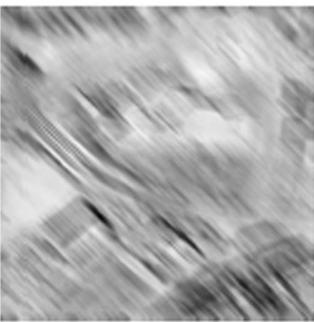

(e)

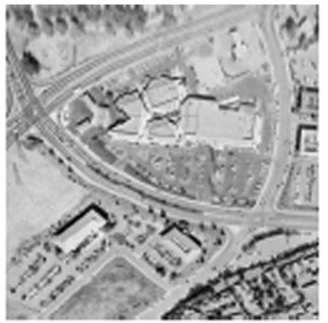

(b)

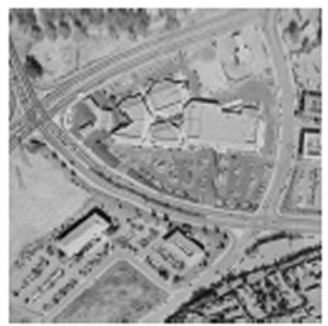

(f)

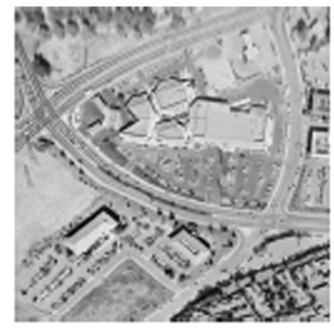

(c)

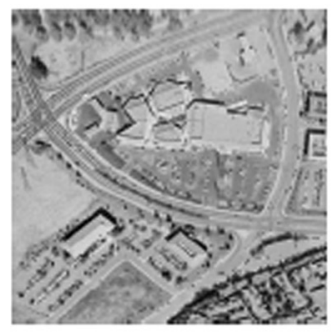

(g)

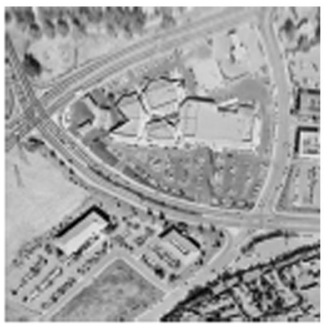

(d)

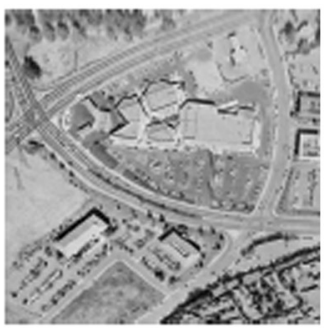

(h)

Fig. 7 Results of noisy images restored by different methods under $55 * 135$ motion blur, with noise level $(\mathrm{a}-\mathrm{d}) \delta=0.02$ and $(\mathrm{e}-\mathrm{h}) \delta=0.1$. Columns from the left to the right in each row are the blurred noisy image, the restored image by FTVd, the restored image by GISA, the restored image by NCHTV-ADMM, respectively. 


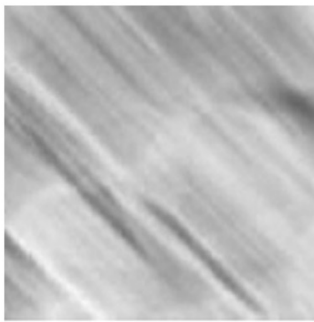

(a)

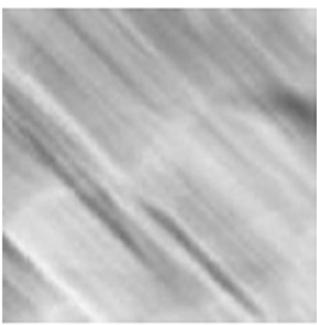

(e)

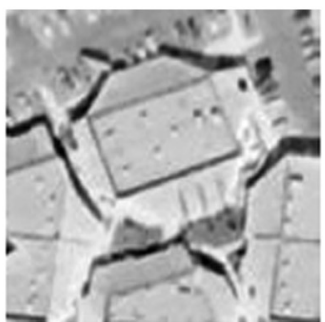

(b)

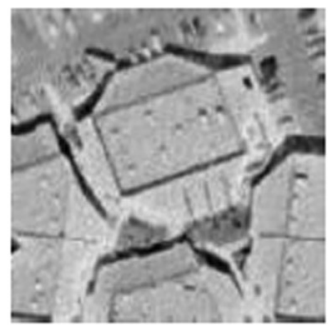

(f)

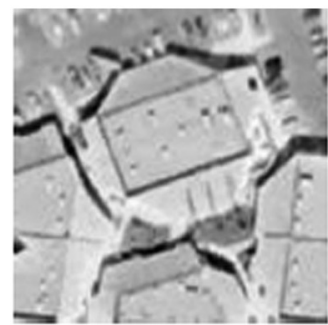

(c)

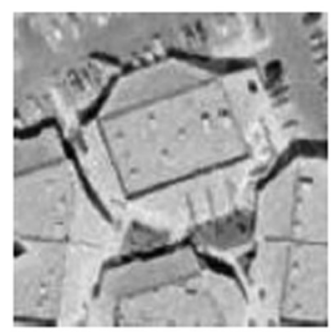

(g)

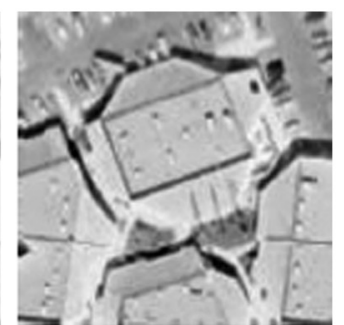

(d)

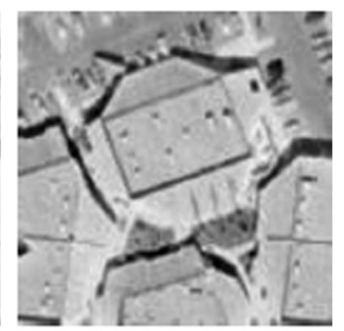

(h)

Fig. 8 Zoomed partial regions in Fig. 7. For a better visualization, some small partial regions of the restored results in Fig. 7 are zoomed.

Table 3 The restored results by FTVd, GISA, and NCHTV-ADMM for different images under motion blur.

\begin{tabular}{|c|c|c|c|c|c|c|c|c|}
\hline \multirow{3}{*}{$\frac{\text { Blurring kernel }}{\text { Motion }(55,135)}$} & \multirow{2}{*}{\multicolumn{2}{|c|}{ Image }} & \multicolumn{3}{|c|}{$\delta=0.02$} & \multicolumn{3}{|c|}{$\delta=0.1$} \\
\hline & & & \multirow{2}{*}{$\frac{F T V d}{31.90}$} & \multirow{2}{*}{$\frac{\text { GISA }}{33.43}$} & \multirow{2}{*}{$\frac{\text { Our method }}{34.57}$} & \multirow{2}{*}{$\frac{\text { FTVd }}{26.03}$} & \multirow{2}{*}{$\frac{\text { GISA }}{26.54}$} & \multirow{2}{*}{$\frac{\text { Our method }}{27.36}$} \\
\hline & Aerial(1) & SNR & & & & & & \\
\hline & & SSIM & 0.9699 & 0.9778 & 0.9827 & 0.8902 & 0.9118 & 0.9326 \\
\hline & Chemical plant & SNR & 29.48 & 30.84 & 32.09 & 23.53 & 24.17 & 25.06 \\
\hline & & SSIM & 0.9334 & 0.9470 & 0.9610 & 0.8114 & 0.8309 & 0.8656 \\
\hline & Aerial(2) & SNR & 36.38 & 37.96 & 39.49 & 28.11 & 29.53 & 31.01 \\
\hline & & SSIM & 0.9337 & 0.9430 & 0.9639 & 0.7754 & 0.8240 & 0.8644 \\
\hline \multirow[t]{6}{*}{ Motion(25,35) } & Aerial(1) & SNR & 34.54 & 35.84 & 37.18 & 28.59 & 28.90 & 29.45 \\
\hline & & SSIM & 0.9839 & 0.9872 & 0.9896 & 0.9407 & 0.9502 & 0.9561 \\
\hline & Chemical plant & SNR & 33.02 & 33.77 & 34.99 & 25.83 & 26.90 & 27.27 \\
\hline & & SSIM & 0.9715 & 0.9746 & 0.9799 & 0.8738 & 0.8992 & 0.9125 \\
\hline & Aerial(2) & SNR & 40.36 & 41.52 & 42.47 & 31.39 & 32.83 & 33.93 \\
\hline & & SSIM & 0.9722 & 0.9754 & 0.9786 & 0.8507 & 0.8861 & 0.9075 \\
\hline
\end{tabular}

\subsection{Experiment 3}

In this subsection, we also perform some experiments to further demonstrate the superiority of our proposed method over FTVd and GISA. We plot three sets of figures to illustrate the convergence performance of the relative errors versus iteration number and SNR versus iteration 
Zhu, Li, and Hao: Restoration of remote sensing images based on nonconvex constrained high-order...

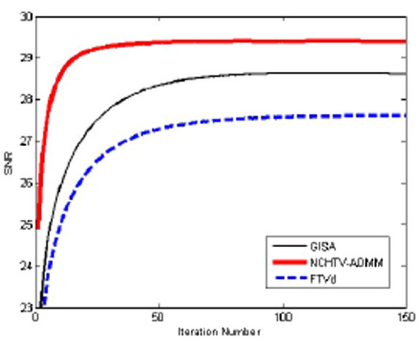

(a) Aerial(1)

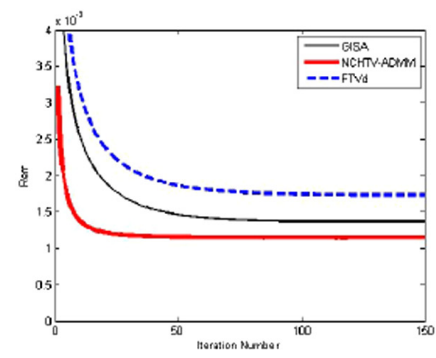

(d) Aerial(1)

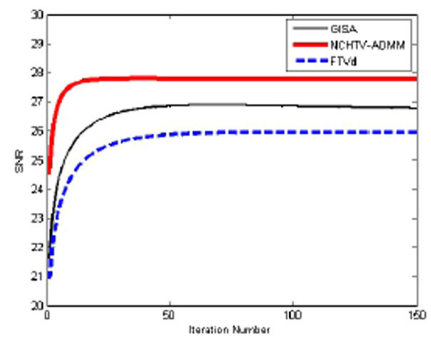

(b) chemical plant

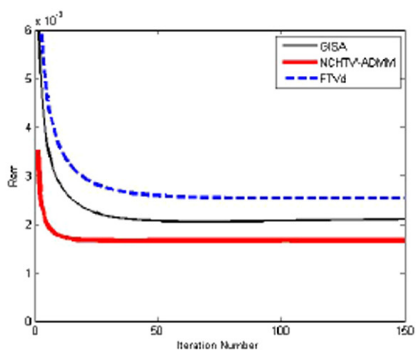

(e) chemical plant

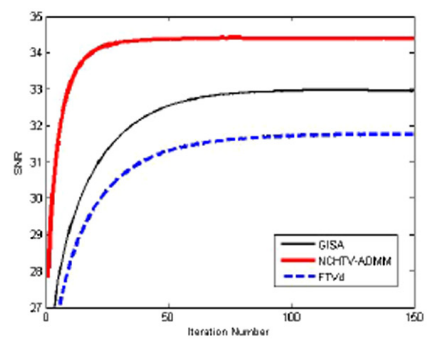

(c) Aerial(2)

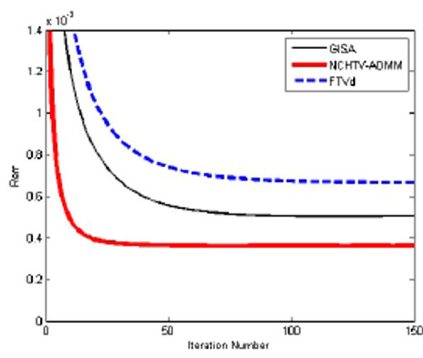

(f) Aerial(2)

Fig. 9 SNR and $R_{\text {err }}$ versus iteration number for three different methods with the noise level $\delta=0.02$ under $11 * 11$ Gaussian blur.

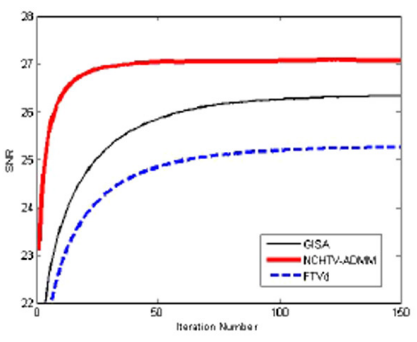

(a) Aerial(1)

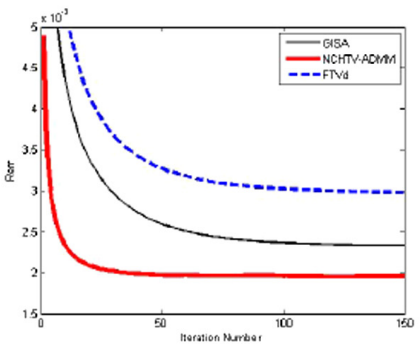

(d) Aerial(1)

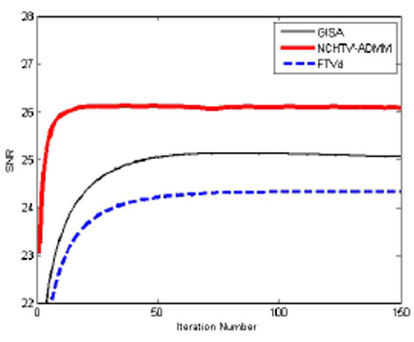

(b) chemical plant

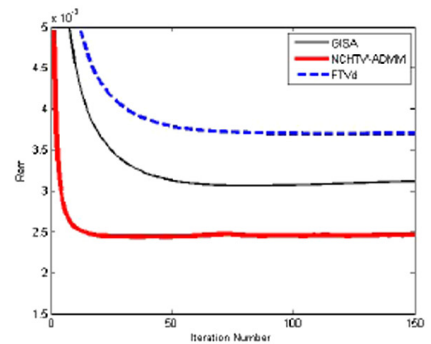

(e) chemical plant

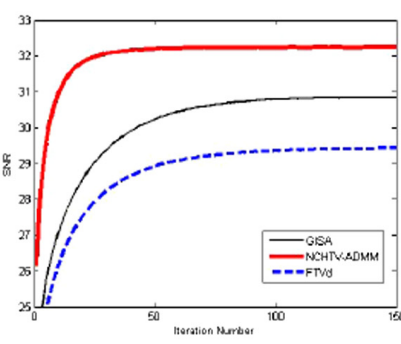

(c) Aerial(2)

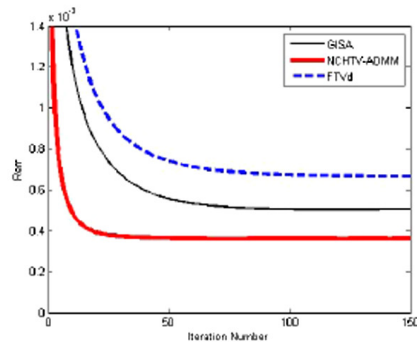

(f) Aerial(2)

Fig. $10 \mathrm{SNR}$ and $R_{\text {err }}$ versus iteration number for three different methods with the noise level $\delta=0.02$ under $15 * 15$ average blur.

number, and the results are shown in Figs. 9-11. As is clearly shown, FTVd, GISA, and our proposed NCHTV-ADMM generate increasing sequences in terms of the iteration number over the SNR, and generate decreasing sequences in terms of the iteration number over the relative errors. Moreover, we find that our proposed method outperforms FTVd and GISA, in terms of highest SNR and lower $R_{\text {err }}$ in fewer iterations. These facts also indicate that the proposed method performs better than FTVd and GISA. 


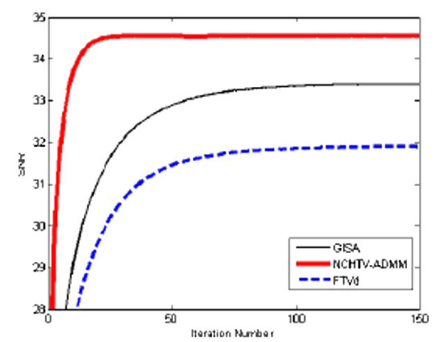

(a) Aerial(1)

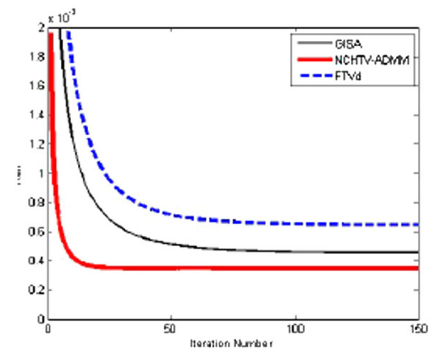

(d) Aerial(1)

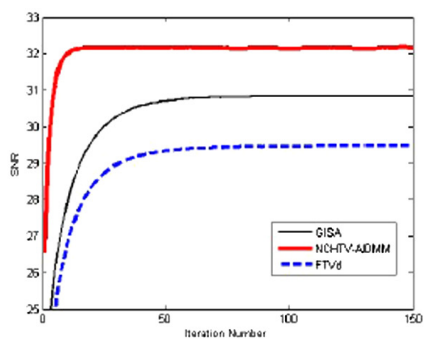

(b) chemical plant

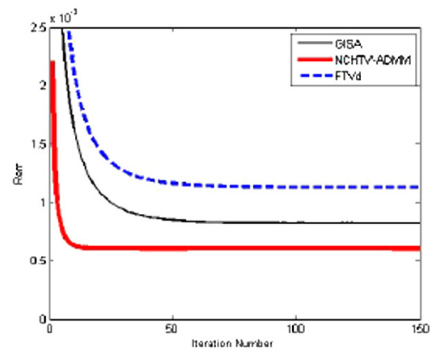

(e) chemical plant

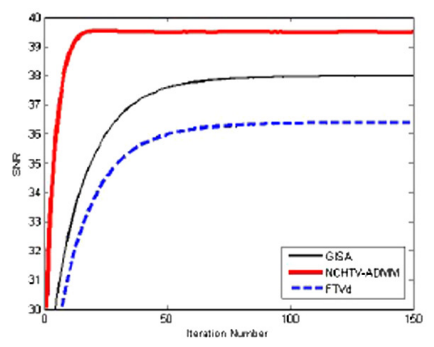

(c) Aerial(2)

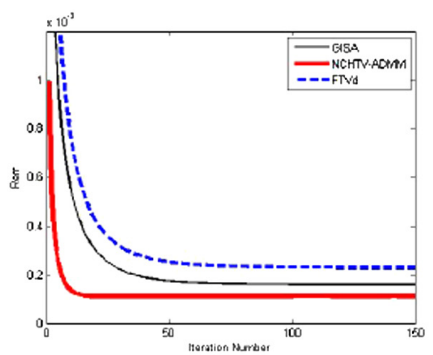

(f) $\operatorname{Aerial}(2)$

Fig. 11 SNR and $R_{\text {err }}$ versus iteration number for three different methods with the noise level $\delta=$ 0.02 under $55 * 135$ motion blur.

\section{Conclusion}

In this paper, we proposed a constrained second-order nonconvex TV regularization image restoration model. A new alternating minimization algorithm that combines generalization of softthresholding algorithm and alternating direction method is proposed to solve the proposed model. Numerical results show that the new proposed model can preserve the edge information while avoiding the staircase effect. By comparison with FTVd and GISA, our proposed method can obtain better performance.

\section{Acknowledgments}

This work was supported by the Training Program of the Major Research Plan of National Science Foundation of China under Grant No. 91746104, the National Science Foundation of China under Grant Nos. 61101208 and 11326186, the Qindao Postdoctoral Science Foudation, China (2016114), a Project of Shandong Province Higher Educational Science and Technology Program, China (J17KA166), Joint Innovative Center for Safe and Effective Mining Technology and Equipment of Coal Resources, Shandong Province of China and SDUST Research Fund (2014TDJH102). The authors declare no conflict of interest.

\section{References}

1. J. Ma and L. Dimet, "Deblurring from highly incomplete measurements for remote sensing," IEEE Trans. Geosci. Remote Sens. 47(3), 792-802 (2009).

2. Y. Chen et al., "Denoising of hyperspectral images using nonconvex low rank matrix approximation,” IEEE Trans. Geosci. Remote Sens. 55(9), 5366-5380 (2017).

3. G. Lianru et al., "Study on the method for estimating the noise in remote sensing images based on local standard deviations," J. Remote Sens. 11(2), 201-208 (2007).

4. X. Wang, H. Zhang, and F. Li, "A PDE-based hybrid model for denosing remote sensing image with Gaussian and salt-pepper noise," Acta Geod. Cartographica Sin. 39(3), 283-288 (2010).

5. F. Xu et al., "Denoising of hyperspectral image using low-rank matrix factorization," IEEE Geosci. Remote Sens. Lett. 14(7), 1141-1145 (2017). 
Zhu, Li, and Hao: Restoration of remote sensing images based on nonconvex constrained high-order...

6. H. F. Shen et al., "Blind restoration of remote sensing images by a combination of automatic knife-edge detection and alternating minimization," Remote Sens. 6(8), 7491-7521 (2014).

7. A. N. Tikhonov and V. Y. Arsenin, Solution of Ill-Posed Problems, Winston, New York (1977).

8. L. I. Rudin, S. Osher, and E. Fatemi, "Nonlinear total variation based noise removal algorithms," Phys. D: Nonlinear Phenom. 60, 259-268 (1992).

9. L. I. Rudin and S. Osher, "Total variation based image restoration with free local constraints," in Proc. of the IEEE Int. Conf. Image Processing (ICIP), Vol. 1, pp. 31-35 (1994).

10. C. R. Vogel and M. E. Oman, "Iterative methods for total variation denoising," SIAM J. Sci. Comput. 17(1), 227-238 (1996).

11. T. F. Chan and P. Mulet, "A nonlinear diffusivity fixed point method in total variation based image restoration," SIAM J. Numer. Anal. 20(2), 1964-1977 (1999).

12. J. G. Zhu and B. B. Hao, "A new noninterior continuation method for solving a system of equalities and inequalities," J. Appl. Math. 2014, 1-6 (2014).

13. Y. M. Huang, M. K. Ng, and Y. W. Wen, "A fast total variation minimization method for image restoration," SIAM J. Multiscale Model. Simul. 7(2), 774-795 (2008).

14. Z. Z. Feng, L. Fang, and G. He, "An iteration primal-dual path-following method, based on wide neighbourhood and large update, for second-order cone programming," Optimization 63(5), 679-691 (2014).

15. J. Tang et al., "A smoothing Newton method for the second-order cone complementarity problem," Appl. Math. 58(2), 223-247 (2013).

16. L. Sun et al., "An accurate active set newton algorithm for large scale bound constrained optimization," Appl. Math. 56(3), 297-314 (2011).

17. A. Chambolle, "An algorithm for total variation minimization and applications," J. Math. Imaging Vision 20(1-2), 89-97 (2004).

18. Z. L. Tian et al., "An accelerated Jacobi gradient based iterative algorithm for solving sylvester matrix equations," Filomat 31(8), 2381-2390 (2017).

19. J. Yu et al., "A decomposition method for large-scale box constrained optimization," Appl. Math. Comput. 231(12), 9-15 (2014).

20. M. Li, X. Kao, and H. Che, "Relaxed inertial accelerated algorithms for solving split equality feasibility problem," J. Nonlinear Sci. Appl. 10(8), 4109-4121 (2017).

21. Y. W. Wen, M. K. Ng, and W. K. Ching, "Iterative algorithms based on decoupling of deblurring and denoising for image restoration," SIAM J. Sci. Comput. 30(5), 2655-2674 (2008).

22. F. Zheng, C. Han, and Y. Wang, "Parallel SSLE algorithm for large scale constrained optimization," Appl. Math. Comput. 217(12), 5377-5384 (2011).

23. C. Y. Han et al., "Parallel algorithms for large-scale linearly constrained minimization problem," Acta Math. Appl. Sin. Engl. Ser. 30(3), 707-720 (2014).

24. R. Y. Zhang, F. F. Xu, and J. C. Huang, "Reconstructing local volatility using total variation," Acta Math. Sin. Engl. Ser. 33(2), 263-277 (2017).

25. X. Lu, H. X. Wang, and X. Wang, "On Kalman smoothing for wireless sensor networks systems with multiplicative noises," J. Appl. Math. 2012, 203-222 (2012).

26. T. Goldstein and S. Osher, "The split Bregman method for L1-regularized problems," SIAM J. Imaging Sci. 2, 323-343 (2009).

27. B. B. Hao and J. G. Zhu, "Fast L1 regularized iterative forward backward splitting with adaptive parameter selection for image restoration," J. Visual Commun. Image Represent. 44, 139-147 (2017).

28. Y. Wang et al., "A new alternating minimization algorithm for total variation image reconstruction," SIAM J. Imaging Sci. 1(3), 248-272 (2008).

29. T. Chan, A. Marquina, and P. Mulet, "High-order total variation-based image restoration," SIAM J. Sci. Comput. 22(2), 503-516 (2000).

30. X. G. Lv, Y. Z. Song, and S. X. Wang, "Image restoration with a high-order total variation minimization method," Appl. Math. Modell. 37, 8210-8224 (2013).

31. J. G. Zhu, K. Li, and B. B. Hao, "Image restoration by a mixed high-order total variation and $l_{1}$ regularization model," Math. Probl. Eng. 2018, 1-13 (2018). 
32. Z. Ren, C. He, and Q. Zhang, "Fractional order total variation regularization for image super-resolution," Signal Process. 93(9), 2408-2421 (2013).

33. Z. Wang, "A numerical method for delayed fractional-order differential equations," J. Appl. Math. 2013, 1-7 (2013).

34. Z. Wang, X. Huang, and J. P. Zhou, "A numerical method for delayed fractional-order differential equations: based on GL definition," Appl. Math. Inf. Sci. 7(2), 525-529 (2013).

35. C. Jiang, F. Zhang, and T. Li, "Synchronization and antisynchronization of N-coupled fractional-order complex systems with ring connection," Math. Methods Appl. Sci. 41(7), 2625-2638 (2018).

36. K. Papafitsoros and C. B. Schönlieb, "A combined first and second order variational approach for image reconstruction," J. Math. Imaging Vision 48(2), 308-338 (2014).

37. K. Bredies, K. Kunisch, and T. Pock, "Total generalized variation," SIAM J. Imaging Sci. 3(3), 492-526 (2010).

38. C. L. Wu and X. C. Tai, "Augmented Lagrangian method, dual methods, and split Bregman iteration for ROF, vectorial TV, and high order models," SIAM J. Imaging Sci. 3(3), 300-339 (2010).

39. A. Parekh and I. Selesnick, "Convex denoising using non-convex tight frame regularization," IEEE Signal Process. Lett. 22(10), 1786-1790 (2015).

40. P. Y. Chen and I. W. Selesnick, "Group-sparse signal denoising: Non-convex regularization, convex optimization," IEEE Trans. Signal Process. 62(13), 3464-3478 (2014).

41. M. Nikolova, M. K. Ng, and C. P. Tam, "Fast nonconvex nonsmooth minimization methods for image restoration and reconstruction," IEEE Trans. Image Process. 19(12), 3073-3088 (2010).

42. X. G. Lv, Y. Z. Song, and F. Li, "An efficient nonconvex regularization for wavelet frame and total variation based image restoration," J. Comput. Appl. Math. 290, 553-566 (2015).

43. H. Zhang et al., "Nonconvex and nonsmooth total generalized variation model for image restoration," Signal Process. 143, 69-85 (2018).

44. Q. Lyu et al., "A comparison of typical $l_{p}$ minimization algorithms," Neurocomputing $\mathbf{1 1 9}$, 413-424 (2013).

45. Z. B. Xu et al., " $l_{\frac{1}{2}}$ regularization: a thresholding representation theory and a fast solver," IEEE Trans. Neural Networks 23, 1013-1027 (2012).

46. W. M. Zuo et al., "A generalized iterated shrinkage algorithm for non-convex sparse coding," in Proc. IEEE Int. Conf. on Computer Vision, Sydney, Australia, pp. 217-224 (2013).

47. M. V. Afonso, J. M. Bioucas-Dias, and M. A. Figueiredo, "An augmented Lagrangian approach to the constrained optimization formulation of imaging inverse problems," IEEE Trans. Image Process. 20, 681-695 (2011).

48. S. Chen, D. Donoho, and M. Saunders, "Atomic decomposition by basis pursuit," SIAM J. Sci. Comput. 20, 33-61 (1998).

49. M. V. Afonso, J. M. Bioucas-Dias, and M. A. Figueiredo, "Fast image recovery using variable splitting and constrained optimization," IEEE Trans. Image Process. 19(9), 2345-2356 (2010).

50. P. Ochs et al., "On iteratively reweighted algorithms for nonsmooth nonconvex optimization in computer vision," SIAM J. Imaging Sci. 8, 331-372 (2015).

51. Z. Lu, Y. Zhang, and J. Lu, " $l_{p}$ Regularized low-rank approximation via iterative reweighted singular value minimization,” Comput. Optim. Appl. 68(3), 619-642 (2017).

52. M. J. Lai, Y. Y. Xu, and W. T. Yin, "Improved iteratively reweighted least squares for unconstrained smoothed lp minimization," SIAM J. Numer. Anal. 51, 927-957 (2013).

53. Y. Peng et al., "Reweighted low-rank matrix recovery and its application in image restoration," IEEE Trans. Cybern. 44(12), 2418-2430 (2014).

54. M. K. Ng, R. H. Chan, and W. C. Tang, "A fast algorithm for deblurring models with neumann boundary conditions," SIAM J. Sci. Comput. 21(3), 851-866 (1999).

55. M. K. Ng and R. J. Plemmons, "Plemmons, fast recursive least squares adaptive filtering by fast fourier transform-based conjugate gradient iterations," SIAM J. Sci. Comput. 17(4), 920-941 (1996).

56. Z. Wang et al., "Image quality assessment: from error visibility to structural similarity," IEEE Trans. Image Process. 13(4), 600-612 (2004). 
Zhu, Li, and Hao: Restoration of remote sensing images based on nonconvex constrained high-order...

Jianguang Zhu is an associate professor at Shandong University of Science and Technology. He received his $\mathrm{PhD}$ in applied mathematics from Xidian University, Xi'an, China, in 2011. His current research interests include optimization theory, algorithms, and applications in image processing and sparse representation.

Kai Li is currently pursuing his MS degree with the College of Mathematics and Systems Science, Shandong University of Science and Technology, Qingdao, China. His current research interests include optimization algorithms in image processing.

Binbin Hao received her PhD in applied mathematics from Xidian University, Xi' an, China, in 2010. She joined the College of Science, China University of Petroleum in 2009, and has been an associate professor since 2012. Her current research interests include image processing, pattern recognition, wavelet analysis, and sparse representation. 